\title{
Functional redundancy of extracellular matrix protein 1 in epidermal differentiation
}

\author{
S. Sercu, Y. Poumay, ${ }^{\star}$ F. Herphelin, ${ }^{\star}$ J. Liekens, L. Beek, $\dagger+$ A. Zwijsen, $\dagger+$ V. Wessagowit, $\S$ D. Huylebroeck, $\dagger \dagger$ \\ J.A. McGrath§ and J. Merregaert \\ Laboratory of Molecular Biotechnology, Department of Biomedical Sciences, University of Antwerp, Universiteitsplein 1, 2610 Wilrijk/Antwerp, Belgium \\ *Cell and Tissue Laboratory, URPHYM, University of Namur, Namur, Belgium \\ †Laboratory of Molecular Biology (Celgen), Department for Molecular and Developmental Genetics, VIB, Leuven, Belgium \\ †aboratory of Molecular Biology (Celgen), Center for Human Genetics, K.U. Leuven, Leuven, Belgium \\ §Genetic Skin Disease Group, St John’s Institute of Dermatology, Division of Genetics and Molecular Medicine, The Guy's, King's College and St Thomas' School \\ of Medicine, King's College London, London, U.K. \\ -Institute of Dermatology, Bangkok, Thailand
}

\section{Summary}

Key words

epidermal differentiation, extracellular matrix

protein 1, lipoid proteinosis, transgenic mice

Conflicts of interest

None declared.
Background Extracellular matrix protein 1 (ECM1) is a secreted protein expressed in skin. Its dermatological relevance has been highlighted by the discovery of loss-of-function mutations in ECM1 in patients with lipoid proteinosis (LiP).

Objectives To determine the role of ECM1 in epidermal differentiation by examining gene and protein expression of epidermal differentiation markers in individuals with LiP and histological assessment of transgenic mouse skin that overexpresses Ecm1a in basal or suprabasal epidermis.

Methods Subconfluent, confluent and postconfluent LiP and control keratinocyte cultures were analysed by Northern and Western blotting for differences in expression of differentiation markers. Expression of these markers was analysed in skin of patients with LiP by immunohistochemistry. To study effects of Ecm1 overexpression on epidermal differentiation, transgenic mice were generated under control of either a keratin 14 or an involucrin promoter.

Results No differential expression of the different markers analysed was observed in LiP keratinocytes compared with controls. No histological differences were found in Ecm1-overexpressing mouse skin compared with wild-type.

Conclusions Absence of ECM1 does not lead to differences in epidermal differentiation. Moreover, overexpression of Ecm1a in vivo does not exert dramatic effects on epidermal structure. Collectively, these findings suggest no role of ECM1 in epidermal differentiation.
Extracellular matrix protein 1 (ECM1) is a secreted glycoprotein involved in diverse biological processes including skin differentiation. ${ }^{1}$ In 2002, loss-of-function mutations in the ECM1 gene were identified in subjects with lipoid proteinosis (LiP). ${ }^{2}$ However, the mechanisms through which the mutations lead to excessive basement membrane deposition in skin and mucosae, bullous skin eruption and atrophic scars are poorly understood. ECM1 encodes four splice variants: ECM1a, expressed in basal keratinocytes, dermal blood vessels and adnexal epithelia including hair follicles and glands; ECM1b, which lacks exon 7 and is expressed in the epider- mal spinous and granular layers; ${ }^{1,3}$ ECM1c, found in the basal layer of the epidermis; ${ }^{4}$ and a variant that generates a truncated protein of 57 amino acids. ${ }^{5}$ One-third of patients with LiP have mutations in exon 7 resulting in loss of ECM1a but not ECM1b, whereas the remainder fails to express either transcript. Thus, keratinocytes and skin biopsies from LiP patients with different mutations in ECM1 provide a unique resource to assess the role of ECM1a and ECM1b in epidermal differentiation. In addition, we also examined the effects of ECM1 overexpression on epidermal differentiation by generating mice overexpressing Ecmla 
at sites of maximal Ecmla expression [basal cells; keratin 14 (K14) promoter and suprabasal layers; involucrin promoter].

\section{Materials and methods}

\section{Cell culture}

Following informed consent, skin biopsies from seven patients with LiP were used to establish keratinocyte cultures (Table 1). Biopsies were taken from individuals with mutations outside exon 7 (patients 1, 4 and 5) and from patients with mutations within exon 7 (patients 2, 3, 6 and 7). Control human keratinocytes were isolated from normal adult skin. Cultures were established using standard methods in autocrine growth conditions and induced to differentiate by manipulating cell density. ${ }^{6}$

\section{Isolation of total RNA and Northern blotting}

Total RNA was extracted from subconfluent, confluent and postconfluent monolayer cultures and analysed by Northern blotting (NB) as described previously. ${ }^{1}$

\section{Antibodies}

The following antibodies were used: mouse monoclonal antibodies to keratin 1 (K1; clone 34bB4; Vector Laboratories, Peterborough, U.K.), keratin 10 (K10; clone LHP1; Vector Laboratories), K14 (clone LL002; Vector Laboratories), loricrin (AF62; gift of Dr D. Hohl, Lausanne, Switzerland) and involucrin (SY5; gift of Dr F. Watt, London, U.K.); rabbit polyclonal antibodies against keratin 5 (K5; clone BL-18; gift of Dr E.B. Lane, Dundee, U.K.); fluorescein isothiocyanate-labelled antimouse and antirabbit secondary antibodies (Dako, Glostrup, Denmark). Rabbit polyclonal antibodies against human ECM1 (EDF) and rabbit polyclonal antimouse Ecm1 (Rb471) antibodies were purified as previously described. ${ }^{1}$ The Rb471 antibody is raised against the oligopeptide CFQKEAPRPDYLLRP (aa288-aa302). Secondary antibodies were horseradish peroxidase (HRP)-conjugated

Table 1 Patients with lipoid proteinosis used to establish keratinocyte cultures

\begin{tabular}{lll}
\hline Patient & $\begin{array}{l}\text { Mutation } \\
\text { in exon 7 }\end{array}$ & $\begin{array}{l}\text { Mutation } \\
\text { outside exon 7 }\end{array}$ \\
\hline 1 & & 507delT \\
2 & Q276X & \\
3 & Q346X & 243delG \\
4 & & 507 delT \\
6 & & \\
7 & Q276X & \\
\hline
\end{tabular}

antirabbit IgG antibody (Rockland Immunochemicals, Philadelphia, PA, U.S.A.) and antihuman K10 antibody (DE-K10; Dako).

\section{Protein expression in lipoid proteinosis keratinocytes and skin}

Proteins from the keratinocyte cultures were extracted according to Poumay and Pittelkow, ${ }^{6}$ fractionated by $10 \%$ sodium dodecyl sulphate-polyacrylamide gel electrophoresis and transferred onto a nitrocellulose membrane (Amersham, Little Chalfont, U.K.). Following incubation with anti-ECM1 antibodies (EDF, $1: 500$ ) and HRP-conjugated antirabbit IgG antibody, ECM1 was detected with the ECL system (Amersham). After informed consent, skin biopsies of nonlesional skin were taken from two individuals with LiP (one patient homozygous for Q276X and the other homozygous for an 1163-bp deletion that removes exon 9 and 10 from the gene). Site-matched control skin was also studied. Indirect immunofluorescence staining was performed on 5- $\mu \mathrm{m}$ cryosections of skin with antibodies to K1 (diluted 1: 10), K10 (diluted 1 : 20), K5 (diluted 1: 1000), K14 (diluted 1:10), loricrin (diluted 1: 10) and involucrin (diluted $1: 10$ ). Labelling procedures were as described previously. ${ }^{7}$ Fluorescein isothiocyanate-labelled antimouse and antirabbit secondary antibodies were used. Propidium iodide was used as nuclear counterstain.

\section{Generation of transgenic mice overexpressing Ecm1a}

Ecm1a cDNA (AF023694) was subcloned downstream of a 2075-bp human K14 promoter (hK14-Ecm1a), which targets expression to the basal layer of mouse epidermis, the outer root sheath of hair follicles, oral epithelia and oesophagus. ${ }^{8}$ To target expression to differentiating suprabasal layers of the epidermis, Ecm1a was cloned downstream of the $3 \cdot 7-\mathrm{kb}$ human involucrin promoter (hInv-Ecmla). ${ }^{9}$ Both constructs were linearised with NotI. DNA was injected into the male pronucleus of fertilised mouse eggs obtained after superovulation of FVB females and implanted into FVB surrogate mothers according to standard procedures. The offspring was characterised by polymerase chain reaction (PCR) on the DNA of tail biopts and transgene expression was analysed by nested reverse transcription (RT)-PCR (primers available on request).

\section{Assessment of transgenic mouse skin}

Protein expression of Ecm1 in skin of transgenic mice was checked by immunohistochemistry on paraffin slides with Rb471 and the Vectastain ABC kit PK4001 (Vector Laboratories). Epidermal differentiation was assessed by light microscopic examination of back skin biopsies after haematoxylin, eosin and safran staining and incubation with anti-K10. 


\section{Results}

\section{ECM1b is expressed only in postconfluent lipoid proteinosis cultured keratinocytes with a mutation in exon 7}

ECM1a/c transcripts were not detected in the cultured keratinocytes from patients with LiP although ECM1a was clearly detected in control cells. In contrast, the ECM1b transcript was expressed in differentiating confluent and postconfluent keratinocyte cultures from individuals with mutations inside exon 7, but not in LiP keratinocytes bearing a mutation outside exon 7 (Fig. 1).

\section{Epidermal differentiation of lipoid proteinosis keratinocytes reveals no major differences compared with control cells}

The differentiation status of the keratinocyte cultures was determined by NB using cDNA probes for K10, K14 and involucrin. ${ }^{10}$ No significant difference in expression of K10 or K14 was detected in mutant cell cultures compared with control keratinocyte cultures (Fig. 1). However, in patient 1 there was a delayed upregulation of involucrin mRNA in the subconfluent culture compared with that of the control (Fig. 1a). This suggests that in absence of ECM1a/b the differentiation of the keratinocytes could be delayed (Fig. 1a) and analysis of the involucrin protein expression supports this interpretation (Fig. 1b). This observation could not be verified in other patients with mutations outside exon 7 (patients 4 and 5) (Fig. 1c).

\section{Epidermal differentiation in lipoid proteinosis skin reveals no major differences compared with control skin}

When nonlesional skin from individuals with LiP and a normal matched control were examined with various differentiation-associated antibodies, no differences in immunostaining patterns were observed (data not shown). Antibodies to K5 and K14 labelled the basal layer with only minor suprabasal staining. The anti-K1 and anti-K10 antibodies labelled the entire epidermis except the basal cell layer. Involucrin immunoreactivity was present in the granular layer with no evidence of increased, decreased or

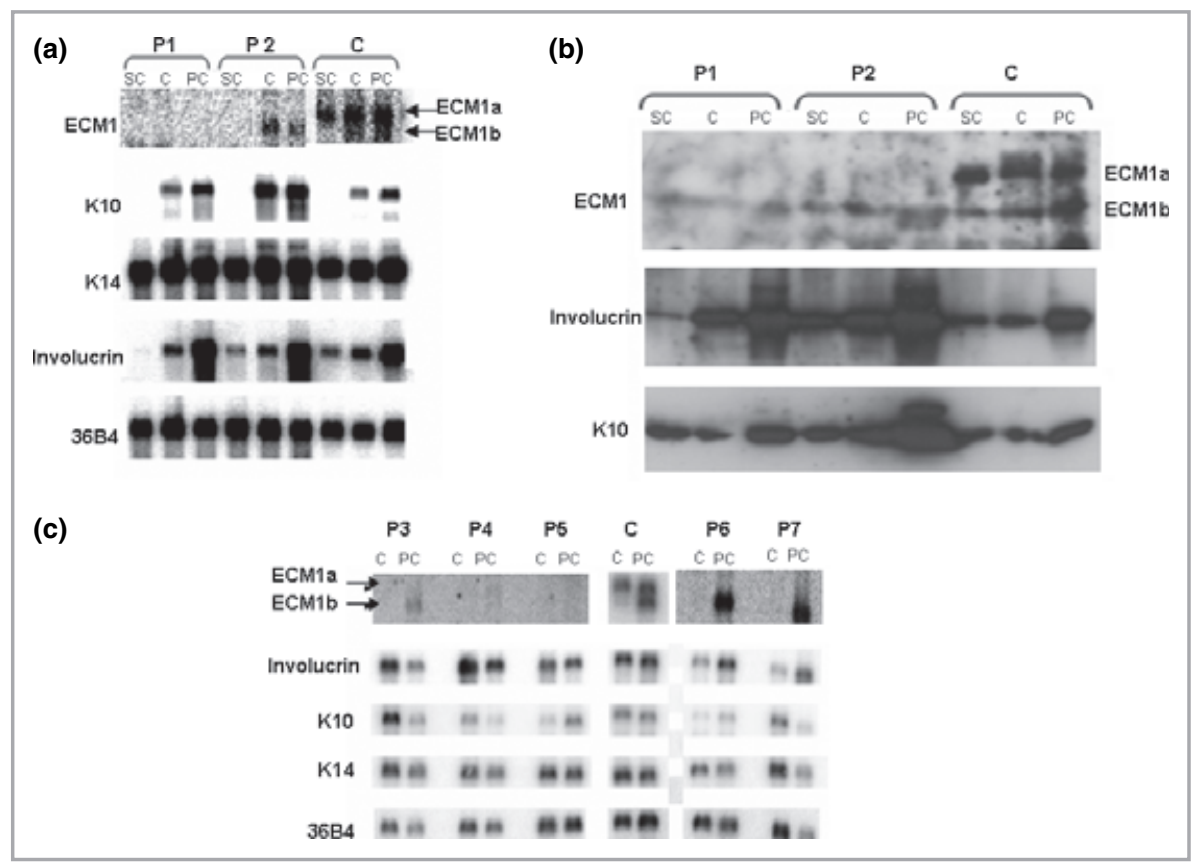

Fig 1. Extracellular matrix protein 1 (ECM1) plays no essential role in the terminal differentiation of human keratinocytes. Human normal (control) and mutant lipoid proteinosis keratinocytes were grown in standard conditions and induced to differentiate. (a) Poly (A+) RNA was extracted from the control (C) and two mutant cell lines [patient 1 (P1) and patient 2 (P2)] when the cells had reached subconfluency (SC), confluency (C) and postconfluency (PC) and was subjected to Northern blot analysis. Blots were hybridised with the full-length human ECM1 cDNA probe. cDNA of different differentiation markers [keratin 10 (K10), keratin 14 (K14) and involucrin] was used to document the proliferation and differentiation state of the cells. RNA loading was checked by hybridisation with the housekeeping gene 36B4. No expression of ECM1a/c was found in the mutant keratinocytes. ECM1b was found only in the confluent and postconfluent state of cells from patient 2 (mutation in exon 7) and the control. (b) Western blot analysis has confirmed the Northern blot results from patients 1 and 2. (c) Similar experiments as described in (a) were performed on patients 3-7 (P3-P7). Human keratinocyte cultures derived from patients (3, 6 and 7) with a mutation in exon 7 still reveal expression of ECM1b. This is in contrast with the absence of any ECM1 transcript in the cell cultures derived from patients with a mutation outside exon $7(4,5)$. 


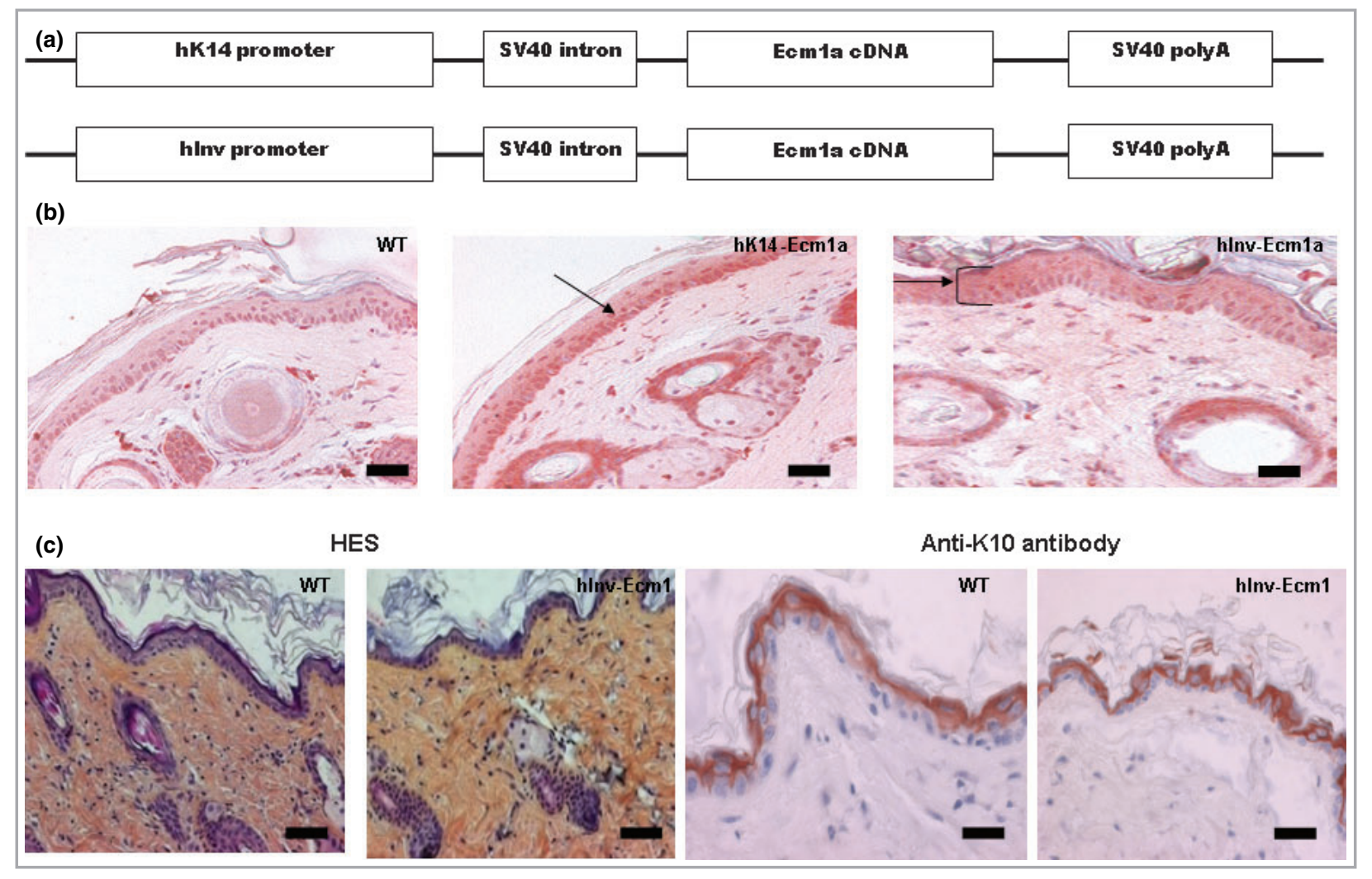

Fig 2. Keratin 14 (K14) and involucrin transgenic mice exhibit normal skin. (a) Transgene cassettes used to generate targeted expression of Ecm 1a under the control of the human basal K14 promoter (hK14) or the human suprabasal involucrin promoter (hInv). (b) Overexpression of Ecm1 was determined by immunohistochemistry with the rabbit-anti-Ecm 1 antibody Rb471. Epidermal differentiation was assessed by light microscopic examination of skin biopsy specimens taken from the tail. Transgenic mice with Ecm1a cDNA under an hK14 promoter revealed Ecm1a overexpression in the basal layer of the skin (arrow), while in transgenic mice with Ecm 1a cDNA under an hInv promoter Ecm1a overexpression was found throughout the whole epidermis (arrow). (c) Morphological analysis of wild-type (WT) and transgenic back skin after haematoxylin, eosin and safran (HES) staining and immunohistochemistry with the antikeratin 10 (K10) antibody. Note normal architecture in all cases. Scale bar represents $100 \mu \mathrm{m}$.

precocious expression in the LiP skin. Similarly, loricrin labelling was present in the superficial granular layer in all skin samples with no increased expression in the stratum corneum.

\section{Epidermal differentiation in transgenic mice overexpressing Ecm1a in basal or suprabasal keratinocytes reveals no major differences compared with wild-type mouse skin}

The in vivo effect of Ecmla on epidermal differentiation was investigated in transgenic mice that overexpress Ecm1a in undifferentiated basal (K14) or differentiated suprabasal layers (involucrin) (Fig. 2a). Increased expression of Ecmla was observed in both transgenic mice by RT-PCR (data not shown) and by immunohistochemistry (Fig. 2b). Targeted expression of Ecmla in differentiated and undifferentiated epidermal layers failed to alter the epidermal morphology from that of wild-type skin (Fig. 2c). These observations indicate that increased expression of Ecmla in vivo does not exert any dramatic effect on epidermal differentiation.

\section{Discussion}

Previous demonstrations of ECM1a expression in the epidermal basal layer and ECM1b in the suprabasal layers have suggested a possible role for ECM1 in terminal keratinocyte differentiation. ${ }^{1}$ This notion has also been supported by the binding characteristics of ECM1 with matrix metalloproteinase (MMP) $-9^{11}$ and perlecan. ${ }^{4}$ MMP-9 is involved in epidermal growth factor-dependent keratinocyte migration. ${ }^{12}$ Perlecan is a large heparan sulphate proteoglycan that is required in the epidermis to maintain keratinocyte homeostasis. ${ }^{13}$

We have shown that ECM1 is not essential for epidermal homeostasis. Keratinocytes from individuals with LiP who harbour a mutation in exon 7 can express ECM1b, in contrast to patients with mutations located elsewhere in the gene. ${ }^{2}$ The presence or absence of ECM1b in LiP skin does not lead to differences in gene or protein expression of early (K10) or late (involucrin) markers of epidermal differentiation. Moreover, the observation that the expression of epidermal differentiation markers was similar to normal skin suggested that lack of ECM1a and of ECM1b has no effect on epidermal maturation. 
Of note, Ecm1a overexpression in the basal (K14) or suprabasal (involucrin) layers of transgenic mice does not change the texture or histological features of the skin on light microscopic analysis.

In conclusion, ECM1 is dispensible for terminal differentiation of keratinocytes in unstressed conditions. In addition, excessive Ecm 1 in mice has no major effects. Additional studies are needed to understand why ECM1 expression and splicing are regulated during the epidermal maturation process.

\section{Acknowledgments}

This work was supported by a grant from the Fund for Scientific Research - Flandres (FWO-G.0133.05) to J.M. and D.H. and partly by grant 2.4506.01 from FRFC to Y.P. S.S. is a fellow of the Instituut voor Wetenschappelijk en Technologisch Onderzoek. We thank Dr T. van de Putte, Dr L. Umans and Dr P. Smits for their help and expertise in creating the transgenic mice.

\section{References}

1 Smits P, Poumay Y, Karperien M et al. Differentiation-dependent alternative splicing and expression of the extracellular matrix protein 1 gene in human keratinocytes. J Invest Dermatol 2000; 114:718-24.

2 Hamada T, McLean WH, Ramsay M et al. Lipoid proteinosis maps to $1 \mathrm{q} 21$ and is caused by mutations in the extracellular matrix protein 1 gene (ECM1). Hum Mol Genet 2002; 11:833-40.

3 Sander CS, Sercu S, Ziemer M et al. Expression of extracellular matrix protein 1 (ECM1) in human skin is decreased by age and increased upon ultraviolet exposure. Br J Dermatol 2006; 154:218-24.
4 Mongiat M, Fu J, Oldershaw R et al. Perlecan protein core interacts with extracellular matrix protein 1 (ECM1), a glycoprotein involved in bone formation and angiogenesis. J Biol Chem 2003; 278:17491-9.

5 Horev L, Potikha T, Ayalon S et al. A novel splice-site mutation in ECM-1 gene in a consanguineous family with lipoid proteinosis. Exp Dermatol 2005; 14:891-7.

6 Poumay Y, Pittelkow MR. Cell density and culture factors regulate keratinocyte commitment to differentiation and expression of suprabasal K1/K10 keratins. J Invest Dermatol 1995; 104: 271-6.

7 Kennedy AR, Heagerty AH, Ortonne JP et al. Abnormal binding of an anti-amnion antibody to epidermal basement membrane provides a novel diagnostic probe for junctional epidermolysis bullosa. Br J Dermatol 1985; 113:651-9.

8 Vassar R, Rosenberg M, Ross S et al. Tissue-specific and differentiation-specific expression of a human K14 keratin gene in transgenic mice. Proc Natl Acad Sci USA 1989; 86:1563-7.

9 Carroll JM, Albers KM, Garlick JA et al. Tissue- and stratum-specific expression of the human involucrin promoter in transgenic mice. Proc Natl Acad Sci USA 1993; 90:10270-4.

10 Watanabe R, Wu K, Paul P et al. Up-regulation of glucosylceramide synthase expression and activity during human keratinocyte differentiation. J Biol Chem 1998; 273:9651-5.

11 Fujimoto N, Terlizzi J, Aho $\mathrm{S}$ et al. Extracellular matrix protein 1 inhibits the activity of matrix metalloproteinase 9 through highaffinity protein/protein interactions. Exp Dermatol 2006; 15:3007.

12 McCawley LJ, Li S, Benavidez M et al. Elevation of intracellular cAMP inhibits growth factor-mediated matrix metalloproteinase-9 induction and keratinocyte migration. Mol Pharmacol 2000; 58:145-51.

13 Sher I, Zisman-Rozen S, Eliahu L et al. Targeting perlecan in human keratinocytes reveals novel roles for perlecan in epidermal formation. J Biol Chem 2006; 281:5178-87. 